\title{
La bicicleta en el país de la (auto)movilidad eléctrica
}

\section{The Bicycle in the land of electric (auto)mobility}

\author{
Edorta Bergua Jiménez ${ }^{1}$ \\ Fecha de recepción: 02-01-2020 - Fecha de aceptación: 03-07-2020 \\ Hábitat y Sociedad (ISSN 2173-125X), n. ${ }^{\circ}$ 13, noviembre de 2020, pp. 107-124. \\ http://dx.doi.org/10.12795/HabitatySociedad.2020.i13.07
}

\section{Summary}

Electric mobility has had a great impact as a major axis of intervention to move forward to a landscape at once less dependent on fossil fuels and more sustainable. Starting out from this framework, the objective we wish to pursue is to draw attention to the restrictive reading imposed on the concept of electric mobility, predominately identified with its automotive branch, leaving aside the bicycle.

The contribution of the electric automobile to the fulfilment of the objectives demanded by sustainable mobility has been tackled, thus demonstrating the limitations of technological solutions and emphasising the need to reduce the number of cars, their usage and speed, and the need to prioritise pedestrians, cyclists and public transport.

After analysing the evolution, benefits and risks of the electric bicycle, it is confirmed that this is by far the most widespread electric vehicle, despite the tiny amount of public aid it receives. It stands out for its powerful strategic value to attract many people to cycling who are reluctant to use conventional bicycles, or who want to make longer journeys effortlessly, or are put off by steep slopes, or want to carry loads or children.

Finally, greater public attention is advocated for electric bicycles, suggesting criteria and lines of action to give shape to policies in this area.

\section{Key words}

Bicycle; Electric mobility; Public policies; Sustainability

\section{Resumen}

La movilidad eléctrica ha irrumpido como un importante eje de intervención para avanzar hacia un escenario menos dependiente de los combustibles fósiles y más sostenible. Partiendo de este marco, la meta perseguida es llamar la atención sobre la lectura restrictiva que se impone del concepto de movilidad eléctrica, identificada predominantemente con su vertiente automovilística, y que ignora a la bicicleta.

Se aborda la aportación del automóvil eléctrico al cumplimiento de los objetivos postulados por la movilidad sostenible, evidenciando las limitaciones de las soluciones tecnológicas e insistiendo en la necesidad de reducir el número de coches, su uso y velocidad; y de primar a los peatones, ciclistas y al transporte público.

Tras analizar la evolución, beneficios y riesgos de la bicicleta eléctrica, se constata que esta es el vehículo eléctrico inmensamente mayoritario, pese a las escasas ayudas públicas que recibe. Se destaca su potente valor estratégico para atraer al ciclismo a muchas personas reacias al uso de la bicicleta convencional, o que desean realizar sin esfuerzo recorridos más largos, por pendientes pronunciadas o transportar cargas o niños.

Finalmente se propugna una mayor atención pública a las bicicletas eléctricas, sugiriendo criterios y líneas de actuación para perfilar las políticas en este ámbito.

\section{Palabras clave}

Bicicleta; Movilidad eléctrica; Políticas públicas; Sostenibilidad 


\section{Introducción}

Desde ya hace tiempo la movilidad eléctrica ha sido presentada como una solución tecnológica que aspira a dar respuesta a los problemas medioambientales originados por el actual modelo de transporte (en particular, su contribución al cambio climático), caracterizado por una elevada proporción de desplazamientos en vehículos particulares propulsados por combustibles derivados del petróleo.

Así planteada, la movilidad eléctrica constituiría uno de los principales ejes de intervención para avanzar hacia un escenario progresivamente menos dependiente del petróleo, que genere menores emisiones contaminantes y sea, por tanto, más sostenible y saludable. Este planteamiento, sin embargo, no se plantea reducir las dimensiones del parque automovilístico, de motocicletas y ciclomotores, ni a cuestionar su carácter eminentemente privado, haciendo además abstracción de los considerables impactos medioambientales, territoriales, urbanos, sociales y en la salud que seguirían acarreando estos vehículos.

Como recientemente ha concluido la Agence nationale de sécurité sanitaire, de l'alimentation, de l'environnement et du travail (2019), a partir de una revisión sistemática de la literatura epidemiológica y toxicológica existente, aunque las mejoras tecnológicas del parque de vehículos lograran una disminución más o menos acentuada de la contaminación atmosférica, estas serán insuficientes por sí solas para mejorar la calidad del aire en las áreas urbanas si no van acompañadas de una reducción del tráfico y de acciones de promoción de la movilidad activa y del transporte colectivo. Ello es debido a que los neumáticos y frenos de los vehículos eléctricos, y el desgaste del asfalto que provocan, son fuentes importantes de emisiones de micropartículas.

Esta sustitución de los motores de combustión por otros eléctricos, aun en el supuesto de que resultara beneficiosa en su balance medioambiental, de ningún modo resuelve las externalidades negativas que provoca este modelo en diversos ámbitos, como son los problemas de salud vinculados al sedentarismo que agrava el uso cotidiano de coches y motos; la siniestralidad vial que causa; los costes económicos y ambientales derivados de la construcción y conservación de sus infraestructuras asociadas; la ocupación, deterioro y fragmentación del territorio que origina; sus afecciones en la calidad de vida y en el bienestar urbano; la contaminación acústica y visual que conlleva; y su ineficacia a efectos de reducir el volumen y la longitud de los desplazamientos que se realizan en estos vehículos.

Sobre la base de los pretendidos beneficios medioambientales del coche eléctrico son cuantiosos y crecientes los recursos que las administraciones públicas vienen destinando a promover su venta y uso. Por el contrario, los programas de ayudas para la adquisición de ciclos de asistencia eléctrica ${ }^{2}$ son con creces más escasos y dotados con presupuestos notablemente inferiores, aun cuando sean los vehículos eléctricos que más ventas registran y mayores beneficios medioambientales ofrecen.

Ante esta paradójica situación, a la vez que preocupante desde una perspectiva de sostenibilidad, este artículo pretende, en primer lugar, alertar sobre esta nueva apuesta a favor del coche como vehículos hegemónico en la movilidad cotidiana. Asimismo, centraremos la atención en los diversos beneficios, ventajas y riesgos que presenta la movilidad ciclista eléctrica, en su relevante auge actual y futuro, y en consecuencia, reclamar para ella una mayor atención pública, proponiendo finalmente algunos criterios y líneas de actuación. 


\section{El impulso de la automoción eléctrica}

La potente apuesta por el coche eléctrico a la que actualmente asistimos responde a una estrategia de la industria automovilística para afrontar el declive de la automoción propulsada por combustibles fósiles, apoyada en el desarrollo tecnológico de los vehículos eléctricos y su progresivo abaratamiento.

En realidad los coches eléctricos existen desde hace casi dos siglos. Su declive se inició en la segunda década del siglo xx, debido al gran impulso del coche con motor de combustión interna, gracias a su mayor radio de acción y elevada velocidad que posibilitaron los avances tecnológicos y la cada vez mayor disponibilidad de gasolina que conllevó el descubrimiento de grandes reservas de petróleo.

La crisis que sufre el sector automovilístico, más allá del impacto negativo que le puede originar la actual fase de desaceleración de la economía global, es consecuencia de tres grandes factores:

a) El ineludible encarecimiento del precio del petróleo, derivado del aumento de la demanda mundial y del mayor costo de producción, en comparación con el de las reservas de hidrocarburos líquidos explotadas anteriormente.

b) Las cada vez mayores exigencias públicas de reducción de las emisiones de $\mathrm{CO}_{2}$ equivalentes de estos vehículos.

Los límites que el Parlamento Europeo aprobó en 2013 respecto a la media de las emisiones de todos los coches vendidos por cada fabricante en 2020 (no superiores a 95 gramos de $\mathrm{CO}_{2}$ por kilómetro), se han endurecido aún más en 2019, de manera que no podrán rebasar los 65 gramos en 2030 (Cordero, 2019).

c) Las previsiones de prohibición a medio plazo de la circulación de vehículos propulsados por motores de combustión.

España, Francia, Irlanda, Noruega, Países Bajos, Reino Unido y Suecia, ya han anunciado prohibir la venta de vehículos de combustión, incluidos los híbridos, para el período 2030-2040 (Cinco Días, 2019).

Esta apuesta industrial va acompañada de un discurso retóricamente ecologista, orientado a que los consumidores sustituyan sus coches por otros eléctricos, pretendidamente no contaminantes, pero sin entrar a cuestionar en ningún momento la hegemonía del automóvil en el actual modelo de movilidad individual motorizada, ni aspirar a reducir las dimensiones del parque automovilístico.

En este contexto, la actitud predominante entre las administraciones públicas se ha caracterizado por prestar apoyo económico, infraestructural, investigador, normativo y comunicativo a la movilidad eléctrica automovilística, ignorando en gran medida el papel que, en la transición hacia una mayor sostenibilidad energética, cabría desempeñar la bicicleta eléctrica, vehículo infinitamente más saludable, ecológico, convivencial y económico. Es más, en su acepción institucional, el concepto de "movilidad eléctrica" se aplica para referirse eminentemente al automóvil eléctrico (englobando, en ocasiones, también a ciclomotores, motocicletas y autobuses eléctricos).

Así, el Gobierno alemán entre 2009 y 2013 destinó cerca de 1400 millones de euros a ayudas para la investigación y desarrollo de automóviles eléctricos; y en 2016 lanzó un plan de subvenciones de casi 900 millones de euros, incluyendo ayudas a su compra (Haubold, 2016). 
Por su parte, el Reino Unido invirtió más de 800 millones de libras esterlinas hasta 2010 (Behrendt, 2018); y el Gobierno español, mediante el Plan Estratégico de Apoyo Integral al Sector de Automoción, prevé destinar 2634 millones de euros para apoyar el "proceso de transición hacia un nuevo modelo de movilidad sostenible, conectada e inteligente", y así los consumidores puedan "elegir el coche que quieran comprar en función de la movilidad que deseen" (Ministerio de Industria, Comercio y Turismo, 2019).

Además, a estas ayudas públicas hay que añadir las que se otorgan para la instalación de estaciones de recarga eléctrica, las cesiones de espacio público para ello, las exenciones de tasas de aparcamiento, la autorización de circular por los carriles-bus, exoneraciones fiscales, etc.

Estos cuantiosos subsidios al coche eléctrico benefician principalmente a los hogares con mayor poder adquisitivo, al ser su precio, aún subvencionado, con creces superior al del automóvil convencional y, por tanto, no accesible a la mayoría de la población.

Por otra parte, en el plano comunicativo las administraciones públicas contribuyen activamente a reforzar y legitimar esta nueva estrategia automovilística presentándola como "ecológica", "sostenible" o "verde" con la intención de lograr una amplia aceptación ciudadana.

A ello hay que añadir la acción legislativa que se impulsa mediante la aprobación de normativas en el ámbito de las políticas urbanas y territoriales, adaptadas al futuro electroautomovilístico que se vislumbra.

\section{¿Es realmente ecológico el coche eléctrico?}

Las ventajas del automóvil eléctrico respecto del convencional, en lo que se refiere a su menor impacto medioambiental en el tráfico urbano, están ampliamente aceptadas, puesto que se admite que durante su circulación se genera menor contaminación acústica, de gases y partículas.

Sin embargo, al margen de estas ventajas circunscritas a las áreas urbanas, desde distintos organismos internacionales se pone en cuestión el pretendido carácter ecológico del coche eléctrico, como pasamos a exponer brevemente:

a) Se considera incorrecto restringir el balance ambiental y energético de los automóviles eléctricos y de sus baterías exclusivamente a su fase de uso, ya que se generan elevadas emisiones de efecto invernadero y contaminantes, así como consumos energéticos $^{3}$ tanto durante su proceso de fabricación, como tras su posterior tratamiento al final de su vida útil (European Environment Agency, 2018a).

Mediante una evaluación rigurosa de las emisiones y consumos energéticos de los coches eléctricos, abarcando la totalidad de su ciclo de vida y el de sus baterías (obtención de las materias primas, fabricación, venta, uso y final de vida), se constata que su eficiencia y beneficios ambientales descienden significativamente (ob. cit.).

b) Una generalización del automóvil eléctrico conllevaría una deslocalización de las emisiones contaminantes (Luengo, 2009), puesto que la mejora en las condiciones medioambientales urbanas iría en detrimento de las de las zonas o países en donde se fabrican. 
c) Las ventajas medioambientales de los coches eléctricos con respecto a los convencionales solo se producen si el mix energético necesario está compuesto principalmente por fuentes de energía renovable, circunstancia esta que a día de hoy no se da en la mayoría de los países.

Así pues, su papel en la reducción de emisiones de gases de efecto invernadero queda supeditado a que se sustituyan las actuales fuentes de producción de energía por otras renovables. Y aún en ese caso, el balance ambiental y territorial también debería imputar los impactos derivados de la construcción y mantenimiento de las nuevas instalaciones de producción energética y sus infraestructuras asociadas, necesarias para abastecer la descomunal demanda de electricidad que conllevaría sustituir el actual parque automovilístico por su equivalente eléctrico.

d) El coche eléctrico, al igual que el convencional, durante su circulación genera partículas finas $\mathrm{PM}_{2,5}$ y partículas $\mathrm{PM}_{10}$, producto del uso de los frenos y de la abrasión de las ruedas contra el asfalto, incrementada por su mayor peso (European Environment Agency, 2018a).

e) Se obvia que la producción de los coches eléctricos y de sus baterías requiere de minerales, tales como el níquel, el litio o el cobalto, cuya obtención implica unos procesos extractivos y de procesamiento contaminantes, al igual que su reciclaje (European Environment Agency, 2018a), y cuyas reservas son limitadas, lo que hace que sea imposible reemplazar todo el parque automovilístico mundial por uno eléctrico, al menos con la actual tecnología de baterías (West, 2017).

f) El carácter ecológico que se le atribuye al coche eléctrico es un factor que paradójicamente podría contribuir a incrementar el volumen y/o la distancia de sus desplazamientos, en la medida en que persuade a sus usuarios de su inocuidad medioambiental y menor consumo energético.

\section{El impacto medioambiental de las bicicletas eléctricas con respecto a los automóviles}

Las bicicletas, al contrario que los coches, no emiten contaminantes a la atmósfera mientras circulan, y durante su ciclo de vida estos son sumamente reducidos en comparación con los de los vehículos motorizados. Además, el proceso de producción de una bicicleta requiere una cantidad enormemente inferior de materiales que un coche, siendo estos más abundantes, económicos y fácilmente reciclables.

Como es evidente, una $\mathrm{BE}^{4}$ tiene un impacto ambiental mayor que el de una convencional, pero este es considerablemente menor que el de los automóviles y motos eléctricas, y se compensa con creces si los desplazamientos en $\mathrm{BE}$ vienen a sustituir a otros realizados en otros vehículos a motor.

Se estima que, en comparación con los coches a gasolina, las emisiones totales de óxidos de nitrógeno, dióxido de carbono y partículas $\mathrm{PM}_{10}$ de las $\mathrm{BE}$ son 59, 39 y 18 veces menores, respectivamente. Además, los costos de energía por $100 \mathrm{~km}$ son 47 veces más altos para estos coches que para las BE (Wachotsch, Kolodziej, Specht, Kohlmeyer y Petrikowski, 2018).
4 En adelante utilizaremos las siglas BE y BC para referirnos a las bicicletas eléctricas y a las bicicletas convencionales, respectivamente. 
Si se comparan las emisiones de gases de efecto invernadero que provoca la fabricación de una batería de una BE (22-30 kg) con los 21,5 $\mathrm{kg}$ de emisiones que se ahorran por cada $100 \mathrm{~km}$ no conducidos en un automóvil de gasolina, se observa que aquellas se equilibran con solo recorrer $100 \mathrm{~km}$ en una BE. ${ }^{5}$

Por otra parte, por cada kilómetro recorrido en BE se ahorran casi $150 \mathrm{~g}$ de emisiones de gases de efecto invernadero, en comparación con un automóvil. Se calcula que en Alemania podrían reducirse los gases de efecto invernadero entre 1,1 y 1,5 millones de toneladas, si se lograra que las BE representaran en 2030 el 35\% de las ventas de bicicletas (Lienhop et al., 2015).

\section{¿En qué cambiaría el actual modelo de movilidad con el coche eléctrico?}

A nuestro juicio, la reflexión sobre el coche eléctrico, más allá de verificar sus aportaciones medioambientales, debería de ir encaminada a tasar su contribución al logro de un modelo urbano y de transporte coherente con los postulados de la movilidad sostenible.

Asumiendo esta perspectiva, podemos concluir que la tecnología eléctrica aplicada a la automoción no contribuye per se a aportar soluciones a los problemas de todo tipo que generan los coches de combustión, ni a transformar el modelo de transporte basado en el uso masivo de estos. El mero reemplazo del actual parque de coches convencionales por eléctricos de ningún modo resuelve las externalidades negativas de este modelo, perpetuándose así los mismos efectos que hoy provoca en la calidad de vida urbana, la salud pública, las arcas públicas, en el territorio y en el medio ambiente.

Como bien sintetiza Andreas Unterstaller, experto en transporte y medio ambiente de la Agencia Europea de Medio Ambiente:

Es muy importante mencionar que ningún coche va a ser nunca $100 \%$ limpio. La llegada del coche eléctrico no va a cambiar eso. Lo que decimos es que si de verdad se necesita un coche, los eléctricos son la mejor opción para el medio ambiente. Sin embargo, para el medio ambiente siempre será muchísimo mejor el transporte público, caminar o ir en bicicleta al trabajo. Un coche siempre va a ser un coche, sustituirlo por otro de otro tipo no va a resolver problemas de transporte tales como la congestión del tráfico (European Environment Agency, 2018b, 4. ${ }^{\circ}$ párrafo).

\section{Innovación y bicicletas eléctricas}

La bicicleta, desarrollo tecnológico del siglo xix, humilde, económico y simple, tiene la virtud de continuar siendo en la actualidad un vehículo perfectamente válido y eficiente para satisfacer gran parte de las necesidades de desplazamiento cotidiano de la población urbana que, recordemos, suelen ser de distancias cortas. ${ }^{6}$ Además, en combinación con los transportes colectivos, permite realizar trayectos de media y larga distancia de puerta a puerta sin necesidad de recurrir al automóvil.

La incorporación de la asistencia eléctrica a su funcionamiento constituye una innovación que está revolucionando el ciclismo en sus diferentes vertientes, y lo va a seguir haciendo, ya que, sin apenas esfuerzo, amplía notablemente su radio de acción, le ayuda a superar con 
facilidad las dificultades orográficas y a transportar cargas más pesadas, posibilitándole así captar un considerable mayor número de viajes y usuarios motorizados, sedentarios o con limitaciones físicas.

$\mathrm{Al}$ igual que el resto de los vehículos, la bicicleta también es un producto que ha ido experimentando una ininterrumpida evolución desde que en siglo xix se inventaran los modelos precursores de lo que hoy se considera una bicicleta convencional. A lo largo de su historia se han ido incorporando múltiples mejoras tecnológicas a las bicicletas iniciales, que han transformado sus mecanismos y accesorios, logrando así que los desplazamientos ciclistas sean más seguros, rápidos y cómodos. Durante este proceso se ha diversificado ampliamente la tipología de ciclos, dando así satisfacción a diferentes necesidades y usos: bicicletas de carreras, de montaña, de carga, híbridas, reclinadas, tándems, plegables, triciclos, bicicletas adaptadas, etc.

Si bien las patentes de las primitivas BE datan de la década de 1890, los primeros prototipos se construyeron en Alemania en 1930, pero su escasa potencia, así como el excesivo peso de sus baterías, motivaron que apenas tuvieran aceptación (Desjardins, 2012).

Curiosamente fue Yamaha, gran empresa japonesa del sector del motor, quién en 1989 inventó el sistema de asistencia eléctrica al pedaleo (Parker, 2002), comercializando sus primeras unidades en 1993. Dado el alto precio de la gasolina en Japón en aquellos años, y el elevado uso de la bicicleta previamente existente, obtuvieron un rápido éxito en este país, llegándose a vender en 1998 más de 200000 BE (CETE Méditérranée, 2006).

Desde entonces el concepto de bicicleta con asistencia eléctrica al pedaleo comenzaría a extenderse velozmente por todo el mundo, de manera que entre 1992 y 1998 se comercializaron más de cincuenta modelos diferentes (Wikipedia, 2019).

El gran incremento de ventas que se produce a partir de la década de 2010 estuvo influenciado, en gran medida, por la irrupción de las baterías de iones de litio, más ligeras y potentes, que vinieron a sustituir a la primera generación de baterías de plomo, y también por las mejoras en las condiciones que inciden en la práctica del ciclismo cotidiano.

\section{La bicicleta eléctrica: el único vehículo eléctrico activo}

La BE presenta una característica fundamental que la diferencia radicalmente del resto de vehículos eléctricos: requerir forzosamente de la tracción muscular del usuario para hacer funcionar su motor. Se trata, pues, del único vehículo eléctrico verdaderamente activo, lo que le convierte en el más beneficioso para la salud de sus usuarios y el medio ambiente.

Sin embargo, no son raros los reproches hacia estas bicicletas por el escaso ejercicio físico que se realizaría, cuestionando así una de las principales virtudes que se le atribuyen al ciclismo.

Las principales investigaciones efectuadas a este respecto refutan estos prejuicios, al demostrar que son similares los niveles de actividad física de los ciclistas eléctricos y de los convencionales. ${ }^{7}$ Este hecho se explica por la confluencia de dos factores:

Si bien es cierto que la actividad física realizada a igual distancia en una $\mathrm{BE}$ es inferior a la que se hace con una convencional, ${ }^{8}$ los ciclistas eléctricos suelen recorrer distancias más largas en sus trayectos en comparación con los ordinarios ${ }^{9} \mathrm{y}$ además utilizan sus bicicletas con mayor frecuencia.
7 Los ciclistas eléctricos realizan 4,463 minutos de Tarea Metabólica Equivalente por semana, frente a los 4,085 de los ciclistas ordinarios. (Castro et al., 2019). Similares conclusiones se recogen en Höchsmann et al. (2018).

8 Un estudio efectuado en Noruega ha demostrado que los usuarios de BE, a igual distancia, realizan un esfuerzo equivalente al $80 \%$ del de los ciclistas ordinarios (Fyhri y Fearnley, 2015).

9 Distintos estudios indican que los viajes en BE suelen ser, aproximadamente, un $50 \%$ más largos que los viajes en BC (Cairns, Behrendt, Raffo, Beaumontd y Kiefere, 2017). 
La dotación de asistencia eléctrica no implica necesariamente que la misma esté siempre en funcionamiento, dado que es su usuario quien opta por activarla (y en qué grado), o no.

Cabría preocuparse, de igual modo, por las eventuales pérdidas netas en la actividad física de los ciclistas eléctricos procedentes de la BC. Aunque pueda sorprender, estas son muy escasas puesto que se compensan por el aumento de la distancia total de los desplazamientos que realizan (Castro et al., 2019).

\section{Ventajas de las bicicletas eléctricas}

Evidentemente, la bicicleta más ecológica, económica y saludable es la convencional, por lo que debería ser la variante a fomentar con prioridad. Con todo, las dotadas de asistencia eléctrica, compartiendo la gran mayoría de las ventajas y beneficios de las BC, presentan una serie de características complementarias que las convierten en la mejor opción ciclista para determinadas personas, necesidades o circunstancias.

\section{Factor crucial para seducir a automovilistas, motoristas y personas con limitaciones físicas}

El motor eléctrico que lleva incorporado, cuando se activa, proporciona una ayuda al pedaleo que exige al ciclista un escaso esfuerzo físico, y la batería que incorpora le presta asistencia eléctrica suficiente para realizar la inmensa mayoría de los desplazamientos cotidianos. Esta menor exigencia representa un factor de gran potencial estratégico a efectos de atraer hacia el ciclismo cotidiano a sectores más amplios (y en especial, a muchos automovilistas y motoristas), que presentan un perfil más sedentario que el del ciclista habitual, y que son reacios al uso de la BC; o a personas con las capacidades físicas mermadas, bien por motivo de edad, enfermedad o discapacidad, que encuentran en el ciclismo eléctrico una alternativa de movilidad activa de otro modo fuera de su alcance.

En este sentido el progresivo envejecimiento de la población europea es un factor que, sin duda, va a contribuir a incrementar las ventas y el uso de las BE.

Muchas de estas personas, al conducir por primera vez una BE comprueban sorprendidas que les resulta factible, agradable y nada fatigoso efectuar gran parte de sus desplazamientos en este vehículo, resquebrajándose así su estereotipo respecto a las exigencias físicas implícitas al pedaleo.

Pruebas de este potencial de atracción las encontramos en un reciente estudio llevado a cabo en el marco del proyecto europeo de investigación PASTA (Physical Activity through Sustainable Transport Approaches) (Castro et al., 2019), a partir de una encuesta a más de 10000 participantes de siete ciudades europeas, que incluía ciclistas convencionales, eléctricos y no ciclistas. El estudio muestra que los viajes realizados en BE substituyen en un $25 \%$ de los casos a otros hechos en automóvil o motocicleta; en un 23\% a viajes en BC; y en un $15 \%$ a viajes en transporte público. Las principales motivaciones para optar por la $\mathrm{BE}$ fueron reducir el esfuerzo físico (26\%), ahorrar tiempo (24\%) y hacer desplazamientos más largos $(24 \%)$.

Otros cuatro estudios confirman que los viajes en BE sustituyen desplazamientos en automóvil en, al menos, un $50 \%$ de los casos (Cairns et al., 2017). 
Es también reseñable el mayor uso de las BE por parte de las mujeres en los Países Bajos, en donde realizan aproximadamente dos tercios del total de desplazamientos en estos vehículos (De Haas, 2019).

\section{Mejoras en la actividad física}

Como ya se ha apuntado, es necesario conceptuar a las BE como vehículos de movilidad activa, dado que requieren de la tracción humana para su funcionamiento, registrando sus usuarios niveles de actividad física similares a los de los ciclistas convencionales.

Distintas investigaciones han demostrado que la práctica regular del ciclismo eléctrico mejora la presión arterial, la capacidad cardiorrespiratoria, el metabolismo de las grasas y el bienestar mental (Höchsmann, 2018).

Además, tiene el valor añadido de lograr que pedaleen con regularidad personas con escasos niveles de actividad física, como las que presentan problemas de sobrepeso, movilidad o de edad avanzada (Dill y Rose, 2012).

\section{Aumento del radio de acción y de la velocidad}

Gracias a la asistencia al pedaleo el radio de acción del ciclista se amplía notablemente, incluso cuando se trata de trayectos por pendientes pronunciadas, bajo fuertes vientos o teniendo que transportar niños o cargas. El estudio de ámbito europeo antes mencionado (Castro et al., 2019) nos muestra que los ciclistas eléctricos urbanos recorren trayectos sensiblemente más largos ( $8 \mathrm{~km}$ de media) que los ciclistas ordinarios $\left(5,3 \mathrm{~km}\right.$ de media),${ }^{10}$ lo cual posibilita sustituir una parte aún mayor de los viajes en automóvil, dado que en la UE el $40 \%$ de estos son de menos de $5 \mathrm{~km}$, y aproximadamente $2 / 3$ menores de $15 \mathrm{~km}$ (Küster, 2017a).

Además, su velocidad media es superior a la de la BC, siendo posible mantenerla estable en prácticamente cualquier circunstancia. Se estima que la velocidad media de una BC en medio urbano oscila entre los 12-15 km/h (Sanz, 1996), mientras que la de la BE está en torno a los 17-20 km/h (Bernardoni, Iseli y Munafò, 2009; y Dozza, Piccinini, y Werneke, 2016).

Ambas cualidades le hacen a la BE ser competitiva con respecto a otros vehículos a motor en muchos recorridos cotidianos, al tiempo que facilita a un amplio abanico de personas la práctica del ciclismo vinculada al turismo, deporte y ocio.

\section{Ayuda al arranque}

La asistencia que proporciona la asistencia eléctrica ayuda al ciclista situado ante un semáforo a arrancar más rápidamente y sin zigzaguear, a fin de distanciarse de otros vehículos, y así ubicarse con mayor comodidad en la calzada o poder realizar un giro con mayor celeridad.

\section{Pilar de la ciclologística}

En el ámbito de los sistemas de logística de última milla, los ciclos eléctricos dotados de dispositivos para el transporte de cargas, constituyen una excelente alternativa a los vehículos motorizados para el reparto de mercancías en áreas urbanas.
10 Cifras oficiales de los Países Bajos confirman esta tendencia, si bien reducen las distancias medias recorridas por ciclistas eléctricos y convencionales $(5,1$ km y 3,5 km, respectivamente) (De Haas, 2019). 
Estos ciclos serían capaces de distribuir el $50 \%$ de las mercancías ligeras y paqueterías en entornos urbanos (European Cyclists' Federation, 2016). Además, son una alternativa real y económica a las flotas de vehículos motorizados de las empresas. Prueba de ello es que en Alemania en 2018 las ventas de ciclos eléctricos de carga crecieron el doble que las de BE ( $80 \%$ y un $36 \%$ respectivamente), superando además las de coches eléctricos (39200 frente a 36062), a pesar de la suculenta subvención de 4000 euros que se concede para la compra de estos (Oltermann, 2019).

\section{Riesgos y desventajas del ciclismo eléctrico}

\section{Siniestralidad}

Como se ha visto, los $250 \mathrm{~W}$ de potencia máxima que puede aportar la asistencia eléctrica a la bicicleta tiene como consecuencia que se registre un diferencial medio de velocidad respecto a las convencionales que oscila en una horquilla entre $2-8 \mathrm{~km} / \mathrm{h}$ superior.

Esta mayor velocidad, que puede suponer una ventaja para ciertos desplazamientos cotidianos y un incentivo para determinados usuarios, es al mismo tiempo un factor de siniestralidad. En efecto, distintos estudios han constatado que los ciclistas eléctricos experimentan más colisiones y casi-colisiones que los ciclistas ordinarios. En estas colisiones resultan afectados mayormente peatones ( $31 \%$ de los eventos críticos), coches y furgonetas $(21 \%)$ y otras bicicletas (18\%) (Dozza, Piccinini y Werneke, 2016).

A esto hay que añadir que el mayor peso de las BE limita su capacidad de reacción y maniobrabilidad ante un imprevisto, y alarga las distancias de frenado, incrementándose así la probabilidad de causar y sufrir un incidente vial.

Sus mayores velocidades provocan con frecuencia que el resto de usuarios de las vías estimen equivocadamente su velocidad real, no siendo capaces de anticipar sus movimientos, al confundirlas con las más previsibles $\mathrm{BC}$.

En cuanto a la gravedad de los accidentes de los ciclistas eléctricos y tradicionales, distintos estudios elaborados en los Países Bajos, Alemania y Suiza, coinciden en no apreciar diferencias significativas entre ambos colectivos si se controla la influencia de la edad (Schepers, Klein y Fishman, 2018).

\section{Conflictos con los peatones}

Si bien las BE no son en sí mismas una nueva fuente de conflicto con los peatones, su creciente presencia incrementa su riesgo comparativamente a las de las BC. Además, al confundirlas con las BC, los peatones estiman incorrectamente su velocidad real, al suponerlas más lentas de lo que son, lo que les lleva a no prever correctamente el ritmo de sus trayectorias.

Las velocidades y el mayor peso de las BE limitan la capacidad de reacción y la maniobrabilidad de estas bicicletas si se produce una situación crítica en la que esté involucrado un peatón. Ambos factores pueden influir, asimismo, en la gravedad de las lesiones que eventualmente les pueden provocar. 


\section{Costo económico}

El rango de precios de las BE es muy amplio, al igual que ocurre con otros tipos de bicicletas, en función de la calidad de sus materiales, fabricante, etc., con el añadido de que su motor y componentes electrónicos (sensores, controlador, display, sistema antirrobo, etc.) encarecen aún más el coste final.

En todo caso, no se suele recomendar la compra de bicicletas urbanas nuevas con asistencia eléctrica por debajo de los 1500-2000 euros, ${ }^{11}$ al considerarse que precios inferiores denotan una calidad dudosa, lo que a la larga supondría mayores costes. Se trata, pues, de cuantías no despreciables, pero aún y todo, muy alejadas del precio de las motos y coches eléctricos. ${ }^{12}$

A ello hay que sumar el importe (en torno a 400 euros) que cada 2-4 años hay que desembolsar para sustituir la batería, el consumo eléctrico de sus correspondientes cargas, así como el superior coste medio de las reparaciones con respecto a las $\mathrm{BC}$, al estar dotadas de dispositivos tecnológicos sofisticados.

\section{Mantenimiento y dependencia técnica}

A las periódicas labores de mantenimiento de los componentes que toda bicicleta requiere, en el caso de las BE hay que añadir las del motor, batería, sistema y componentes electrónicos. La mayor complejidad de estas labores y de las reparaciones generan una mayor dependencia con respecto de los servicios técnicos.

\section{Conversiones de ciclistas convencionales a eléctricos}

El ahorro en términos de esfuerzo físico que proporciona la asistencia eléctrica presenta al mismo tiempo el riesgo de llegar a seducir también a ciclistas convencionales en perfectas condiciones físicas, a utilizar la $\mathrm{BE}$ en recorridos que podrían hacerse con $\mathrm{BC}$ sin dificultad. A la hora de realizar un desplazamiento parece superfluo recurrir a la BE, un vehículo más sofisticado tecnológicamente que la BC, con un mayor impacto ambiental y costo económico, cuando resulta posible hacerlo mediante una solución más simple como es esta última.

Existen algunos indicios que apuntarían en este sentido, como es el hecho de que en los Países Bajos se esté detectando un aumento en la proporción de jóvenes usuarios de $\mathrm{BE}$ para ir a la escuela o a la universidad (Peine, Van Cooten y Neven, 2016).

\section{Ventas de bicicletas y coches eléctricos, y ayudas públicas}

Muestra del potencial de las BE en el parque ciclista internacional es la explosión de sus ventas en Asia y Europa durante la presente década, así como su cada vez mayor cuota de mercado respecto al conjunto de bicicletas vendidas.

En China, en 2004 las ventas de BE rondaron los 7,5 millones de unidades, los 16 millones en 2006 (Fairley, 2005), y los 23 millones en 2013 (Electric Bikes Worldwide Reports, 2013). En la Unión Europea, en 2018 se vendieron más de 2,5 millones de BE, destacando Alemania y los Países Bajos, en donde se vendieron 980000 unidades $(23,5 \%$ del
11 El precio medio de una BE en Alemania (año 2014) era de 2350 euros, menos del $8 \%$ de la media del precio de un automóvil eléctrico (30000€) (Haubold, 2016). El precio medio de las BE vendidas en el Estado español en 2018 fue de 2165 euros (Asociación de Marcas y Bicicletas de España, 2019).

12 A los mayores precios de estos vehículos hay que añadirles el impuesto de circulación, el seguro de responsabilidad civil y los costes de aparcamiento; así como sus superiores costes de mantenimiento, reparación y sustitución de las baterías. 
13 Elaboración propia a partir de datos recogidos en los informes anuales sobre el sector económico de la bicicleta publicados por la Asociación de Marcas y Bicicletas de España durante el periodo 20132018.

14 Estas ayudas se vehicularon a través de distintos Planes de Impulso al Medio Ambiente «PIMA Aire», consistentes en otorgar 200 euros por BE, hasta el agotamiento de los fondos provistos, compartidos para la compra de diferentes vehículos eléctricos (Gobierno de España, 2014).

15 Elaboración propia a partir de datos recogidos en los informes anuales sobre el sector económico de la bicicleta publicados por la Asociación de Marcas y Bicicletas de España.

16 A título de ejemplo cabe mencionar el caso del Gobierno alemán, que entre 2009 y 2013 destinó cerca de 1400 mi-

llones de euros en ayudas a la investigación y desarrollo de automóviles eléctricos, a lo que en 2016 se añadió un plan de subvenciones de casi 900 millones de euros, incluyendo ayudas a la compra. A fecha de 2016, las subvenciones públicas a la compra de BE fueron prácticamente inexistentes. A pesar de ello, se estima que el número de $\mathrm{BE}$ en servicio entonces era de 2,5 millones de unidades, mientras que el de coches eléctricos no superaba los 25500 (Haubold, 2016).

17 Elaboración propia a partir de los datos definitivos anuales de matriculaciones de automóviles publicados por la Asociación Nacional de Importadores de Automóviles, Camiones, Autobuses y Motocicletas.

18 Elaboración propia a partir de datos recogidos en los informes anuales sobre el sector económico de la bicicleta publicados por la Asociación de Marcas y Bicicletas de España durante el periodo 20132018. total de bicicletas comercializadas, y un incremento del $36 \%$ con respecto a 2017 (Van Schaikon, 2019)); y 409 000, respectivamente (40,4\% del total y un aumento del 29\% en relación con 2017 (Bovag, 2019)).

La Confederación de la Industria Europea de la Bicicleta (CONEBI) estima que en 2025 en la UE se alcanzarán unas ventas cercanas a los 7 millones de unidades, cantidad que otros expertos elevan a $10 \mathrm{mi}$ llones (Oortwijnon, 2019).

A estas bicicletas habría que añadir aquellas convencionales que se han electrificado, fórmula más económica y que permite aprovechar la flota ciclista ya existente.

Según las prospecciones de la Federación de Ciclistas Europeos, se espera que el número de $\mathrm{BE}$ en circulación en la UE alcance los $62 \mathrm{mi}-$ llones de unidades para 2030 (Küster, 2017b).

En lo que respecta al Estado español, durante el periodo 2013-2018 se vendieron $275850 \mathrm{BE},{ }^{13}$ cifra que representa el $4,2 \%$ del total de bicicletas comercializadas. Este porcentaje ha ido en progresivo aumento, intensificándose en 2017 y, en especial, en 2018, cuando supusieron el 6,5\% y el 10,6\%, respectivamente, de todas las ventas de bicicletas.

El ciclismo eléctrico en el Estado español, al igual que en muchos países occidentales, ha sido objeto de distintas convocatorias de subvenciones públicas para incentivar su compra. El conjunto del esfuerzo económico del Gobierno español cabe situarlo en torno a los 1,3 millones de euros, y se concentró exclusivamente entre 2013 y $2015 .{ }^{14} \mathrm{Me}-$ diante estas ayudas se subvencionó la compra de, aproximadamente, 6700 unidades, un 12,8\% del total de BE comercializadas en ese periodo, lo que supone el 2,4\% de todas las BE vendidas entre 2013 y $2018 .{ }^{15}$

A partir de 2015, solo el Área Metropolitana de Barcelona y el Ente Vasco de la Energía han seguido lanzando líneas de ayuda a la compra de BE, con cuantías entre 200 y 250 euros por unidad.

Estas sorprendentes cifras de ventas de BE alcanzadas en la UE con escasas ayudas gubernamentales, contrastan sobremanera con las del sector de la automoción eléctrica que, contando con un relevante mayor apoyo económico público, ${ }^{16}$ sin embargo han recogido magros resultados. En efecto, las ventas de BE en Europa superan a las de los automóviles eléctricos en una proporción de 10 a 1, llegando en China a ser de 20 a 1 (Bruce, 2019). Cabe afirmar, por tanto, que la bicicleta es, sin parangón, la verdadera protagonista de la movilidad eléctrica, aun cuando el relato dominante ignore deliberadamente esta contundente realidad.

Esta misma tendencia internacional se confirma igualmente en el Estado español, en donde durante el periodo 2013-2018 se contabilizaron 27224 matriculaciones de automóviles eléctricos, ${ }^{17}$ frente a las $275850 \mathrm{BE}$ vendidas, ${ }^{18}$ lo que significa que estas representan más de 9 de cada 10 vehículos eléctricos comercializados.

\section{Conclusiones y recomendaciones para abordar el ciclismo eléctrico en las políticas de movilidad}

En la actualidad las políticas de movilidad están alineadas con la estrategia de mera sustitución de los coches de combustión por otros provistos de motores eléctricos, que impulsa el sector automovilístico como solución pretendidamente ecológica a los problemas medioambientales que aquéllos generan. 
El optimismo tecnológico subyacente a esta estrategia va dirigido a transmitir la idea de que esos problemas tienen solución mediante avances técnicos, ocultando las consecuencias medioambientales y sociales que originan los procesos de producción, uso y final de vida de los coches eléctricos. Este planteamiento, además, no ofrece solución alguna a los impactos de todo tipo que seguiría acarreando el uso masivo de automóviles y motos eléctricas, puesto que no está orientado a reducir su volumen, ni la longitud de sus desplazamientos.

El papel de las administraciones públicas ante esta potente apuesta por el coche eléctrico se ha distinguido por prestarle un sólido apoyo y por reducir conceptualmente la movilidad eléctrica a su vertiente automovilística y, en menor medida, al transporte público, destinando muy escasos recursos públicos a la promoción de la BE. Esta minusvaloración del ciclismo eléctrico resulta paradójica con respecto al discurso oficial que propugna una transición hacia un modelo de mayor sostenibilidad energética, dado que la $\mathrm{BE}$ es el vehículo eléctrico con diferencia más vendido, saludable, ecológico, convivencial y económico de todos, y el único que es activo y capaz de atraer hacia el ciclismo cotidiano a amplios sectores de usuarios de coches, motos, y/o personas de perfil sedentario o con problemas vinculados a la movilidad personal. Todas estas razones justificarían que la BE mereciera una atención pública sustancialmente mayor y se le otorgara un rango estratégico en el marco de las políticas de movilidad ciclista.

En la otra cara de la moneda, no hay que desdeñar el mayor impacto ambiental de las $\mathrm{BE}$ con respecto a las convencionales, así como el incremento de la siniestralidad y de los conflictos con los peatones que sus mayores velocidades pueden provocar, y que también afectarían a la convivencia y al deterioro de la seguridad y de la calidad peatonal.

Consideramos que los avances esenciales hacia un modelo de movilidad más sostenible no van a venir de la mano de soluciones tecnológicas, sino de afrontar decididamente una reducción sustancial del número de coches, de su uso, velocidad y de la oferta para su estacionamiento; acompañada de fórmulas para su uso compartido; de una apuesta prioritaria a favor de los viajes a pie, en bicicleta y en transporte público; y de políticas orientadas a la reducción de las necesidades de desplazamiento y de las distancias a recorrer.

Desde esta perspectiva, las políticas públicas deben tomar conciencia del relevante papel que la bicicleta desempeña en el ámbito de la movilidad eléctrica, para así dar respuesta a sus necesidades y condicionantes, muchos de ellos compartidos con el ciclismo urbano convencional. Obviamente, esta posición favorable al ciclismo eléctrico debe venir acompañada de una actitud cautelosa dirigida a evitar o minimizar lo más posible los potenciales riesgos señalados.

Partiendo de estas premisas pasamos a sugerir una serie de criterios y líneas de actuación que podrían ser de utilidad a la hora de perfilar las políticas a desarrollar en el ámbito del ciclismo eléctrico:

1) Las posibilidades de desarrollo del ciclismo eléctrico están estrechamente condicionadas por la existencia de entornos urbanos cómodos y seguros para la movilidad ciclista, sin los cuales no cabe esperar que pueda prosperar de modo significativo. Consecuentemente, las políticas en el ámbito del ciclismo eléctrico, para que sean eficaces y coherentes, deben estar incardinadas en una planificación ciclista integral. 
2) El fomento público de la BE no debe plantearse de modo indiscriminado y tendría que evitar dirigirse a la población físicamente activa que no requiere de la asistencia eléctrica para desplazarse en bicicleta. Convendría que esta promoción se centrara en ofrecer la posibilidad de probarla e incentivar su uso, a aquellos sectores reacios al uso de la $\mathrm{BC}$, como son los automovilistas, motoristas y personas con escaso nivel de actividad física y/o que presentan problemas de movilidad o son de edad avanzada.

3) Siendo la experimentación personal un factor clave para la promoción de la $\mathrm{BE}$, uno de los ejes prioritarios de actuación debe ser ofrecer al público la posibilidad de probar aquellas BE más adaptadas a sus necesidades, ${ }^{19}$ en colaboración con el sector del comercio ciclista, a fin de dar a conocer personalmente sus ventajas y prestaciones. Estos programas incluirían un periodo de prueba durante un tiempo limitado a cambio de un alquiler, con posterior derecho a compra, ofreciendo también la opción de electrificar la bicicleta propia.

Estos programas deberían de estar dotados con presupuestos equiparables, al menos, a los destinados a la adquisición de coches y motos eléctricas, atendiendo a su elevada efectividad para avanzar hacia la descarbonización del sistema de transporte.

Por otra parte, si bien las BE pueden ser la mejor opción ciclista para determinadas personas, necesidades o circunstancias, para la mayoría las BC resultan ser las más adecuadas y económicas. Por consiguiente, sería justo que en paralelo se lanzaran líneas de subvenciones, de igual o mayor cuantía, para la adquisición de todo tipo de bicicletas no eléctricas adecuadas para la movilidad cotidiana.

4) A la vista del previsible incremento del número de BE y de que sus velocidades aumentan el riesgo de conflicto y la siniestralidad con los peatones, la normativa y la planificación viaria deberían establecer instrumentos que garanticen la seguridad y el confort de estos, incidiendo particularmente en la regulación de la velocidad, y en el establecimiento de restricciones de acceso, cuando la intensidad peatonal así lo exija.

5) Este incremento del ciclismo eléctrico, derivado del aumento previsto del parque ciclista y de su mayor utilización en comparación con las $\mathrm{BC}$, exigirá una ampliación de las redes viarias ciclistas, de modo que puedan ser capaces de absorber el aumento de sus flujos. Además, implicará mayores radios de curvas y de las secciones, ya que sus velocidades más elevadas, particularmente en las aceleraciones que se producen durante los adelantamientos, requerirán que estos se efectúen manteniendo una distancia superior.

6) El aumento del radio de acción que ofrecen las BE y el incremento de los flujos ciclistas previsto son factores añadidos que deberían contribuir a incentivar la extensión de las vías ciclistas de alta capacidad que, recordemos, permiten los desplazamientos en bicicleta de mayores distancias en un tiempo más reducido, y de manera segura y confortable.

19 Distintas investigaciones sugieren que entre el 30 y el $70 \%$ de las personas que prueban una BE se interesan en la posibilidad de comprar una (Cairns et al., 2017).
7) Dado el superior coste de las BE con respecto al de las BC, su expansión está directamente determinada por la existencia de una oferta suficiente de dotaciones de aparcamiento en origen y destino que garanticen niveles adecuados de protección meteorológica y ante el vandalismo y el robo. 
8) A fin de hacer más competitiva la ciclologística, y poder seguir haciendo uso del viario ciclista, sería preciso que la normativa legal autorizara un aumento de la potencia por encima de los 250 W en los ciclos de pedaleo asistido de carácter comercial destinados a transporte de personas o cargas, manteniendo el requisito de interrumpir la alimentación eléctrica cuando la velocidad supera $\operatorname{los} 25 \mathrm{~km} / \mathrm{h}$. 


\section{Referencias bibliográficas}

Agence nationale de sécurité sanitaire, de l'alimentation, de l'environnement et du travail (2019). Pollution de l'air : nouvelles connaissances sur les particules de l'air ambiant et l'impact du trafic routier. Anses. Recuperado el 7 de diciembre de 2019, de: https:/ /www. anses.fr/fr/content/pollution-de-1\%E2\% 80\%99airnouvelles-connaissances-sur-les-particules-de$1 \%$ E2\% 80\% 99air-ambiant-et-1\%E2\% 80\%99impact.

Asociación de Marcas y Bicicletas de España (2019). El sector de la bicicleta en cifras, 2018. AMBE. Recuperado el 7 de diciembre de 2019, de: http:/ / asociacionambe.es/wp-content/uploads/2019/06/ Presentaci\%C3\%B3n-Nota-de-Prensa-2018_AMBE. pdf.

Behrendt, Frauke (2018). Why cycling matters for electric mobility: towards diverse, active and sustainable e-mobilities. Mobilities, $13, \mathrm{n}^{\circ} 1,64-80$. DOI: 10.1080/17450101.2017.1335463.

Bernardoni, Sebastiano; Iseli, Aude y Munafò, Sébastien (2009). Usagers, usages et potentiel des vélos à assistance électrique vélos à assistance électrique. Résultats d'une enquête menée dans le canton de Genève. Observatoire Universitaire de la Mobilité UNIGE. Ginebra: Université de Genève. Recuperado el 7 de diciembre de 2019 de: https:/ /www.osti.gov/etdeweb/servlets/purl/22123350.

Bovag (2019). Fietsen in de statistiek 2011-2018 Nederland. AJ Bunnik: Bovag. Recuperado el 7 de diciembre de 2019 de: https:/ / www.bovag.nl/BovagWebsite/media/BovagMediaFiles/Cijfers/2019/ Fietsverkoopcijfers-2011-2018.pdf.

Bruce, Oliver (2019, 22 de octubre). If You Care About Climate Change, You Should Care About Micromobility. Micromobility America. Recuperado el 7 de diciembre de 2019 de: https:/ / micromobility.io/ blog/2019/10/22/if-you-care-about-climate-change-you-should-care-about-micromobility.

Cairns, Sally; Behrendt, Frauke; Raffo, David; Beaumontd C.; y Kiefere, C. (2017). Electrically-assisted bikes: Potential impacts on travel behaviour. Transportation Research Part A: Policy and Practice, 103, 327342. DOI: 10.1016/j.tra.2017.03.007.

Castro, Alberto et al. (2019). Physical activity of electric bicycle users compared to conventional bicycle users and non-cyclists: Insights based on health and transport data from an online survey in seven European cities. Transportation Research Interdisciplinary Perspectives, 1. DOI: 10.1016/j.trip.2019.100017.

CETE Méditérranée (2006). Le vélo électrique. Etat de l'art en 2006. Niza: CERTU. Recuperado el 7 de diciembre de 2019 de: http:/ / ertia2.free.fr/Niveau2/ Trouvailles/Velo_electrique_-_Etat_de_1_art_2006. pdf.
Cinco Días (2019, 29 de agosto). Bruselas: "Prohibir la venta de los coches de combustión no es compatible con la UE”. Recuperado el 7 de diciembre de 2019 de: https:/ / cincodias.elpais.com/cinco$\operatorname{dias} / 2019 / 08 / 28 /$ companias/1567020462_781646. htm.

Cordero, Dani (2019, 29 de septiembre). El coche eléctrico se enfrenta a una ruta llena de baches. El País. Recuperado el 7 de diciembre de 2019 de: https://elpais.com/economia/2019/09/27/actualidad/1569576975_608804.html.

De Haas, Mathijs (2019). Het gebruik van de e-fiets en de effecten op andere vervoerwijzen. Den Haag: Kennisinstituut voor Mobiliteitsbeleid. KiM. Ministerie van Infrastructuur en Waterstaat. Recuperado el 7 de diciembre de 2019 de: https:/ /www.kimnet.nl/binaries/kimnet/documenten/rapporten/2019/12/02/gebruik-van-e-fiets-en-effecten-opandere-vervoerwijzen/KiM+Rapport+e-fietsgebruik. def.pdf.

Desjardins, David (2012, 17 de mayo). La vague électrique. Vélo Mag. Recuperado el 7 de diciembre de 2019 de: https:/ /www.velomag.com/actualites/reportage/la-vague-electrique/.

Dill, Jennifer; y Rose, Geoffrey (2012). Electric Bikes and Transportation Policy Insights from Early Adopters. Journal of the Transportation Research Board, 2314(1), 1-6. DOI: 10.3141/2314-01.

Dozza, Marco; Piccinini, Giulio Francesco Bianchi; y Werneke, Julia (2016). Using naturalistic data to assess e-cyclist behavior. Transportation Research, 41(Part F), 217-226. DOI: 10.1016/j. trf.2015.04.003.

Electric Bikes Worldwide Reports (2015). Electric Bikes Worldwide Reports. Recuperado el 7 de diciembre de 2019 de: http:/ /www.ebwr.com/sample-pages /

European Cyclists' Federation (2016). Cycling Logistics: the Future of Goods Delivery. Bruselas: European Cyclists' Federation. Recuperado el 7 de diciembre de 2019 de: https://ecf.com/sites/ecf.com/files/ Factsheet-ITF2012-CLOG.pdf.

European Environment Agency (2018a). Electric vehicles from life cycle and circular economy perspectives. TERM 2018: Transport and Environment Reporting Mechanism (TERM) report. Luxemburgo: Publications Office of the European Union. Recuperado el 7 de diciembre de 2019 de: https:/ /www.eea.europa.eu/ publications/electric-vehicles-from-life-cycle/.

European Environment Agency (2018b, 17 de diciembre). Electric vehicles: a smart choice for the environment. European Environment Agency. Recuperado el 7 de diciembre de 2019 de: https:/ / www.eea.europa. $\mathrm{eu} /$ articles/electric-vehicles-a-smart. 
Fairley, Peter (2005). China's cyclists take charge: Electric bicycles are selling by the millions despite efforts to ban them. IEEE Spectrum, 42(6), 54-59. DOI: 10.1109/MSPEC.2005.1437044.

Fyhri, Aslak; y Fearnley, Nils (2015). Effects of e-bikes on bicycle use and mode share. Transportation Research Part D: Transport and Environment, 36, 45-52. DOI: $10.1016 / \mathrm{j} . t r d .2015 .02 .005$.

Gobierno de España (2014, 28 de febrero). Aprobado el Plan PIMA Aire 3. Madrid: La Moncloa. Recuperado el 7 de diciembre de 2019 de: https:/ /www. lamoncloa.gob.es/consejodeministros/paginas/ enlaces / 280214-planpima3.aspx.

Haubold, Holger (2016). Electromobility for all. Financial incentives for e-cycling. European Cyclists' Federation. Recuperado el 7 de diciembre de 2019 de: https://ecf.com/sites/ecf.com/files/FINAL \%20 for $\% 20$ web $\% 20170216 \% 20 \mathrm{ECF} \% 20$ Report_E\%20 FOR\%20ALL-\% 20FINANCIAL \% 20INCENTIVES\%20FOR\%20E-CYCLING.pdf.

Höchsmann, Christoph et al. (2018). Effect of E-Bike Versus Bike Commuting on Cardiorespiratory Fitness in Overweight Adults: A 4-Week Randomized Pilot Study. Clinical Journal of Sport Medicine, 28(3), 255-265. DOI: 10.1097/JSM.0000000000000438.

Küster, Fabian (2017a, 4 de octubre). Include (electric) bicycle parking in the revision of the EPBD. European Cyclists' Federation. Recuperado el 7 de diciembre de 2019 de: https:/ / ecf.com/sites/ecf.com/files/ITRE\%20vote\%20on\%20EPBD_ECF\%20position \% 20paper.pdf.

Küster, Fabian (2017b, 6 de octubre). 62 million electric bicycles in 2030 in the EU need a home. European Cyclists' Federation. Recuperado el 7 de diciembre de 2019 de: https:/ / ecf.com/news-and-events / news / 62-million-electric-bicycles-2030-eu-need-home.

Lienhop, Martina et al. (2015). Pedelection: Verlagerungsund Klimaeffekte durch Pedelec-Nutzung im Individualverkehr. Endbericht: Institut für Transportation Design (Hochschule für Bildende Künste Braunschweig) / Institut für Energie-und Umweltforschung Heidelberg GmbH. Recuperado el 7 de diciembre de 2019, de: https:/ /www.erneuerbarmobil.de/sites/default/files/2016-09/150916_Abschlussbericht_Pedelection_final.pdf.

Luengo, Fernando (2009). Las deslocalizaciones internacionales. Una visión desde la economía crítica. Instituto Complutense de Estudios Internacionales, Universidad Complutense de Madrid. Working Paper $\mathrm{N}^{\circ}$ 10/09. Recuperado el 7 de diciembre de 2019, de: http:/ / eprints.ucm.es/9557/1/WP_10-09.pdf.

Ministerio de Fomento (2000). Encuesta de Movilidad de los españoles residentes. Movilia 2001. Madrid: Ministerio de Fomento, 2000.

Ministerio de Industria, Comercio y Turismo (2019, de 4 de marzo). Nota de prensa, "Reyes Maroto presenta el Plan Estratégico de Apoyo Integral al Sector de Au- tomoción”. Recuperado el 7 de diciembre de 2019 de: https:/ / www.mincotur.gob.es/es-es/gabineteprensa/notasprensa/2019/documents/190304\%20 np\%20automocion.pdf.

Oltermann, Philip (2019, 25 de agosto). Four wheels bad, but three sehr gut. Germans climb aboard cargo bikes. The Guardian. Recuperado el 7 de diciembre de 2019 de: https:/ / www.theguardian.com/ world/2019/aug/25/cargo-bikes-berlin-four-wheels-bad-transport.

Oortwijnon, Jack (2013, 5 de agosto). Europe's E-Bike Imports Indicate Market Size. Bike Europe. Recuperado el 7 de diciembre de 2019 de: https:/ / www.bikeeu.com/sales-trends/nieuws/2013/08/europes-ebike-imports-indicate-market-size-10110166.

Oortwijnon, Jack (2019, 2 de octubre). Volume European E-Bike Market Forecasted to Triple within Next Five Years. Bike Europe. Recuperado el 7 de diciembre de 2019 de: https://www.bike-eu.com/sales-trends/ nieuws / 2019/10/volume-european-e-bike-marketforecasted-to-triple-within-next-five-years-10136694.

Parker, Alan A. (2002, 2-4 de octubre). The power assisted bicycle: a green vehicle to reduce greenhouse gasemissions and air pollution. 25th Australasian Transport Research Forum. Incorporating the Bureau of Transport and Regional Economics' Transport Colloquium. Canberra, Australia. Recuperado el 7 de diciembre de 2019 de: http:/ / alanparker-pest. org/Publications20002004files/ARTF\%20PABsParker02.pdf.

Peine, Alexander; van Cooten, Vivette; y Neven, Louis (2016). Rejuvenating design: Bikes, batteries, and older adopters in the diffusion of ebikes. Science, Technology, Ẽ Human Values, 42 (3), 429-459. Recuperado el 7 de diciembre de 2019, de: https://journals.sagepub.com/doi/ abs/10.1177/0162243916664589.

Sanz, Alfonso; Pérez, Rodrigo; y Fernández, Tomás (1996). La bicicleta en la ciudad. Manual de políticas y diseño para favorecer el uso de la bicicleta como medio de transporte. Madrid: Ministerio de Fomento.

Schepers, Paul; Klein, Karin; y Fishman, Elliot (2018). The Safety of E-Bikes In The Netherlands, Discussion Paper. París: International Transport Forum / OECD. Recuperado el 7 de diciembre de 2019 de: https:/ / www.itf-oecd.org/sites/default/files/docs/safety-ebikes-the-netherlands_0.pdf.

Van Schaikon, Jan-Willem (2019, 21 de marzo). One Million E-Bikes Sold in Germany in 2018; Up 36 Percent!. Bike Europe. Recuperado el 7 de diciembre de 2019 de: https://www.bike-eu.com/sales-trends/ nieuws/2019/03/one-million-e-bikes-sold-in-germany-in-2018-up-36-percent-10135573.

Wachotsch, Ulrike; Kolodziej, Andrea; Specht, Bernhard; Kohlmeyer, Regina; y Petrikowski, Falk (2014). Electric bikes get things rolling. The environmental impact of pedelecs and their potential. Dessau-Roßlau: (German) Federal Environment Agency (UBA). 
Recuperado el 7 de diciembre de 2019 de: https:/ / www.umweltbundesamt.de/sites/default/files/medien/378/publikationen/hgp_electric_bikes_get_ things_rolling.pdf.

Wikipedia (2019). Vélo à assistance électrique. Recuperado el 7 de diciembre de 2019 de: https:/ / fr.wikipedia.org/wiki/V\%C3\%A9lo_\%C3\%A0_ assistance_\%C3\%A9lectrique.
West, Karl (2017, 29 de julio). Carmakers' electric dreams depend on supplies of rare minerals. The Guardian. Recuperado el 7 de diciembre de 2019 de: https://www.theguardian.com/environment/2017/jul/29/electric-cars-battery-manufacturing-cobalt-mining.

Bergua Jiménez, Edorta (2020). La bicicleta en el país de la (auto)movilidad eléctrica. Hábitat y Sociedad, 13, 107-124.

<http://dx.doi.org/10.12795/HabitatySociedad.2020.i13.07>

$\bigcirc$ 